Journal of

Synchrotron

Radiation

ISSN 0909-0495

Received 18 January 2013

Accepted 5 February 2013

\section{Electron yield soft X-ray photoabsorption spectroscopy under normal ambient-pressure conditions}

\author{
Yusuke Tamenori \\ Japan Synchrotron Radiation Research Institute/SPring-8, 1-1-1 Kouto, Sayo, Hyogo 679-5198, \\ Japan. E-mail: tamenori@spring8.or.jp
}

\begin{abstract}
Ambient-pressure soft X-ray photoabsorption spectroscopy (XAS) was demonstrated to be applicable to the chemical analysis of hydrated transitionmetal compounds. For this purpose, even under ambient-pressure conditions, electron yield detection XAS (EY-XAS), based on a simple drain-current setup, was used to overcome a weakness in fluorescence yield detection XAS (FYXAS), which does not give a pure soft XAS. The feasibility of EY-XAS was investigated and it was clarified that the EY-XAS under ambient-pressure conditions corresponds to the mixed data of the total EY and conversion EY spectra. Normal ambient-pressure EY-XAS analysis was applied to anhydrous $\left(\mathrm{CoCl}_{2}\right)$ and to hydrated $\left(\mathrm{CoCl}_{2} \cdot 6 \mathrm{H}_{2} \mathrm{O}\right)$ cobalt chloride at the $\mathrm{Co} L_{23}$-edge. The present measurements demonstrated the ability to unambiguously distinguish the different chemical states of cobalt ions, relying upon spectral differences that indicate octahedral/quasi-octahedral structural changes as a result of hydration/ dehydration reactions.
\end{abstract}

Keywords: soft X-ray photoabsorption spectroscopy; normal ambient pressure; electron yield detection; conversion electron yield detection; hydration.

\section{Introduction}

$\mathrm{X}$-ray photoabsorption spectroscopy (XAS), which is often called X-ray absorption fine-structure (XAFS) spectroscopy, is one of the best techniques for the determination of the electronic structures of materials (Stöhr, 1992). One of the advantage of XAS analysis is that samples can be analyzed regardless of their physical state (solid, gas or liquid), and it is not restricted to the analysis of certain elements, as are Mössbauer and nuclear magnetic resonance (NMR) analysis. Furthermore, because the core electrons excited by X-ray absorption are localized to the atoms from which they originate, X-ray core-electron transitions allow investigation of the chemical properties of specific elements in complex materials. In particular, XAS analyses using soft X-rays are uniquely appropriate not only for studying light elements but also for studying transition metals (Zaanen et al., 1985; de Groot \& Kotani, 2008). Since XAS is governed by dipole selection rules, the $d$-shell properties of first-row transition metals are best probed by the $L_{23}$-edge absorption ( $2 p-3 d$ transition) in the soft X-ray region.

However, so far the application of soft $\mathrm{X}$-ray analysis has been highly restricted. Since soft X-rays below $2.0 \mathrm{keV}$ are largely absorbed by air under ambient pressure, it is standard procedure to perform soft X-ray analysis under high vacuum. Although the high-vacuum chamber produces an ideally clean environment for the sample, chemically significant phenomena take place under ambient pressure, such as the coexistence of water and oxygen gas. One of the active areas of modern research in soft X-ray analysis is to improve the versatility of the sample environment. For example, vacuumcompatible sample holders containing a liquid cell have been developed for the analysis of liquid materials (Guo et al., 2004; Liu et al., 2007; Schreck et al., 2011) and for the observation of chemical reactions (de Smit et al., 2008; de Groot et al., 2010; Huse et al., 2010; Kolmakov et al., 2012).

Ideally, it would be convenient for researchers to be able to apply soft X-ray analytical techniques to their samples without using high-vacuum chambers and special vacuum-compatible reaction cells. One solution is to use a helium path because helium gas has a higher transmission than air in the soft X-ray region as a result of its smaller atomic number. Since the pioneering work by Roper et al. in 1992, several investigations have been reported to obtain reliable spectra under atmospheric pressure conditions using soft $\mathrm{X}$-rays as a probe (Roper et al., 1992; Yagi et al., 2004; Nakanishi et al., 2010). In this scenario, the high vacuum of the light source or synchrotron radiation beam is protected by isolating the analysis chamber using a vacuum window. For example, Nakanishi et al. (2010) have reported their recent successful XAS analysis of hydrated compounds at the $\mathrm{Mg} \mathrm{K}$-edge region $(1.3 \mathrm{keV})$ using a thin $\mathrm{Be}$ foil $(15 \mu \mathrm{m})$ as a vacuum 
window. However, soft X-ray analysis under atmospheric pressure is still limited to energies higher than $1 \mathrm{keV}$, since photoabsorption by the window reduces the photon flux arriving at the sample. To overcome the current limitations, we developed a new approach to XAS analysis using a differential pump instead of a vacuum window (Tamenori, 2010). We specifically focused on the application of lower-energy soft $\mathrm{X}$-rays (below $1 \mathrm{keV}$ ) under normal ambient-pressure conditions, the range in which the $K$-edges of carbon and oxygen and the $L_{23}$-edges of $3 d$ transition metals are found.

Another issue to be solved is the development of an XAS signal detection technique instead of fluorescence detection. Fluorescence yield (FY) detection, which is the more common XAS signal detection method under high pressure, has two drawbacks. One is the spectral line shape distortion, which is well known as the 'self-absorption effect' (Jaklevic et al., 1977; Zschech et al., 1992; Meitzner \& Fischer, 2002). Another issue is that FY measurements do not produce pure XAS, when multiplet effects are important, because the fluorescence decay probability strongly depends on the final state (de Groot et al., 1994). In contrast to fluorescence decay, the Auger decay probability is approximately constant. Therefore, even under normal ambient-pressure conditions, the application of electron yield (EY) measurements is indispensable to obtaining reliable XAS in the soft X-ray region.

In the present research, we demonstrate the capabilities of normal ambient-pressure soft XAS using EY detection. This technique was used for XAS measurements of the coordination states in hydrated transition-metal compounds. We observed a change in the coordination states of cobalt ions in cobalt(II) chloride via hydration/dehydration using $L_{23}$-edge XAS analysis. Such hydrated compounds adopt different crystal structures from those of their dehydrated counterparts and, consequently, generate distinct XAS. This technique provides information about the symmetry and oxidation state of the analyte and allows for the identification of unknown species, establishing it as a powerful tool for speciation analysis of transition metals under normal ambient-pressure conditions.

\section{Experimental}

\subsection{Soft X-ray source}

The normal ambient-pressure soft XAS set-up was installed at the cbranch of the soft X-ray photochemistry beamline (BL27SU) of SPring-8. Radiation from a figure- 8 undulator was dispersed by a soft X-ray monochromator with varied-line-spacing plane gratings, and then introduced into the XAS instrument (Tanaka et al., 1998; Ohashi et al., 2001). XAS were

Figure 1 obtained by scanning the undulator gap as well as the monochromator to maintain maximum intensity of the incident soft X-rays, and by scanning the width of the entrance and exit slits to maintain constant resolving power. The photon-energy-resolving power was set to $E / \Delta E=5000$. The X-ray beam diameter was focused to a $200 \mu \mathrm{m}$ spot on the sample. During analysis, the intensity of the incoming photon beam was monitored by measuring the drain current on the surface of a post-focusing mirror.

\subsection{Ambient-pressure soft $X$-ray spectroscopy set-up}

A schematic diagram of the experimental set-up is shown in Fig. 1 . The XAS chamber was connected to the beamline via a four-stage differential pump (Tamenori, 2010). When the sample chamber was filled with helium (1 atm), the pressure at the uppermost stage of the differential pump was maintained at $1.0 \times 10^{-4} \mathrm{~Pa}$. The system provided enough isolation between the sample chamber atmospheric pressure and the high vacuum of the beamline across a short distance, eliminating the need for a vacuum window for the soft X-ray beamline. The loss of photon flux in the differential pump section was less than $5 \%$ at $800 \mathrm{eV}$, roughly one-sixth of the loss observed when using a $200 \mathrm{~nm} \mathrm{Si}{ }_{3} \mathrm{~N}_{4}$ vacuum window. The total transmission at $800 \mathrm{eV}$ (at the cobalt $L_{23}$-edge) measured $50 \mathrm{~mm}$ downstream from the last aperture (diameter $0.5 \mathrm{~mm}$ ) was about $90 \%$. Additionally, there was no steep reduction in photon intensity at the absorption edge of the elements contained in the vacuum window, providing ideal conditions for XAS analysis. The pressure of the analysis region could be reduced to $1.0 \times 10^{0} \mathrm{~Pa}$ via evacuation through the aperture of the differential pump, and pure helium gas was installed to produce the normal ambient-pressure conditions.

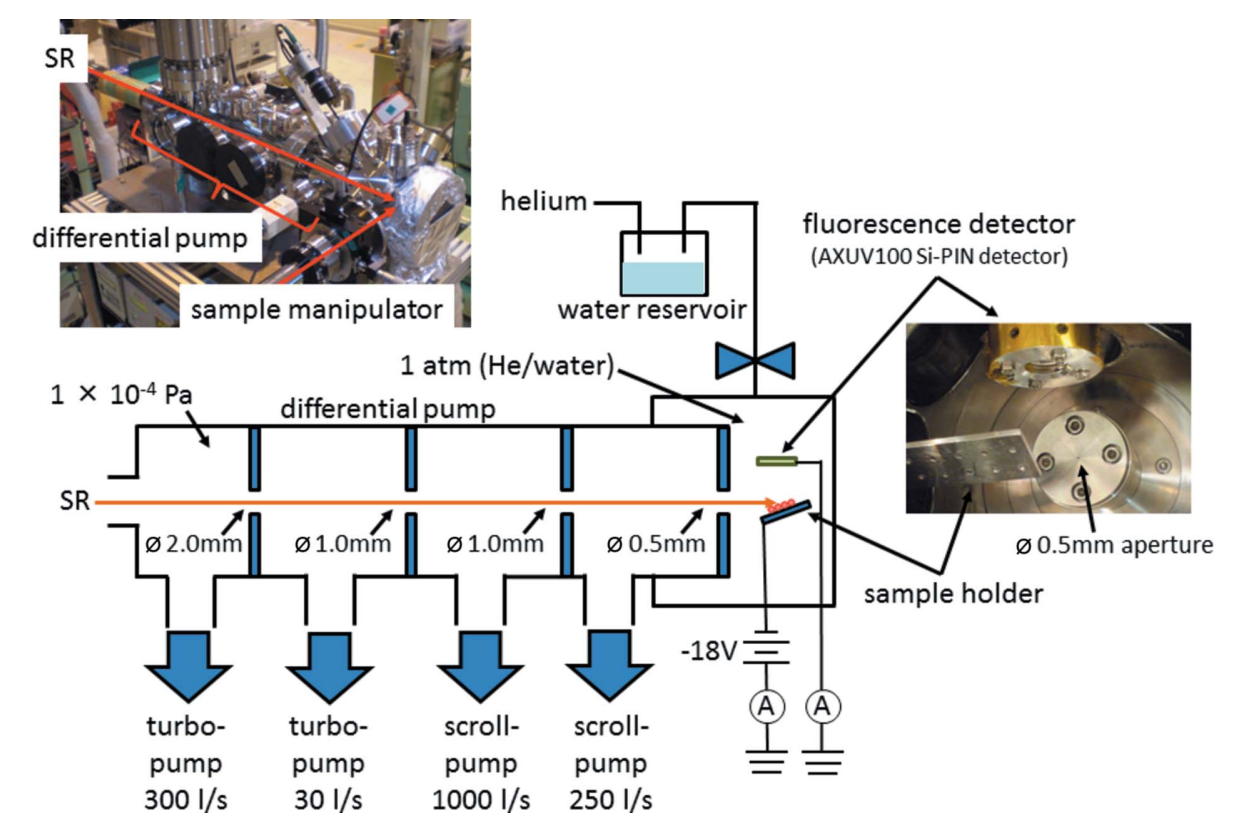

Schematic diagram of the normal ambient-pressure XAS apparatus. Inset photographs show pictures of the whole system (top left) and the inside of the analysis region taken from the downstream of the system (right). 
Evaporation of water from the sample was significantly reduced under normal ambient pressure compared with in a vacuum. However, even under conditions of $1 \mathrm{~atm}$ of helium, drying of the sample could not be prevented completely. Therefore, in order to keep the sample hydrated, a heliumwater mixed gas was introduced into the XAS sample chamber. During analysis of the hydrated samples, helium gas was passed through a reservoir containing purified water at room temperature, and the mixed gas was introduced through a nozzle with an inner diameter of $6.25 \mathrm{~mm}$. The moisture level of the sample chamber was monitored by the color of the $\mathrm{CoCl}_{2}$ sample. Because the hydration/dehydration reaction is fast and is accompanied by a change in color, cobalt chloride is used as a moisture indicator in drying agents (Solomon, 1945). $\mathrm{CoCl}_{2} \cdot 6 \mathrm{H}_{2} \mathrm{O}$ is deep purple in color, whereas the anhydrous form is sky blue (Grime \& Santos, 1934).

\subsection{XAS measurements}

XAS were obtained using both EY and FY detection. EYXAS were recorded using the same experimental set-up as that for conventional sample drain-current measurements. The aluminium sample holder was biased $(-18 \mathrm{~V})$ by two dry batteries $(9 \mathrm{~V})$ with respect to the analysis chamber, and the drain current was monitored by an ammeter. The FY-XAS were recorded using a Si photodiode detector (AXUV-100; IRD), which was installed in front of the sample (Fig. 1). EYXAS and FY-XAS were recorded simultaneously for a sequence of incident $\mathrm{X}$-ray energies in the usual fashion used for XAS measurements. Monochromatic light was irradiated at an angle of $\sim 45^{\circ}$ relative to the sample normal. Data analysis to remove the background and qualitatively analyze the XAS was carried out manually. The data were normalized for variations in the primary X-ray intensity. A linear pre-edge was removed for each spectrum and the data were normalized by the height of the edge jump.

\subsection{Sample preparation}

In the present research, we chose a colored silica gel ball as a sample, since it is easier to check the color of the sample and to control hydrating/dehydrating conditions than it is using pure $\mathrm{CoCl}_{2}$ powder. Anhydrous colored silica gel $\left(\mathrm{CoCl}_{2} /\right.$ $\mathrm{SiO}_{2}$ ) was purchased from Hakuyo. The concentration of $\mathrm{CoCl}_{2}$ in the silica gel was $4 \%$. The hydrated sample was prepared by immersing the anhydrous sample in purified water for $1 \mathrm{~h}$. Purified water was obtained using a water purification system (Barinsted EASYpure RF).

As a standard material, high-purity cobalt(II) chloride hexahydrate $\left(\mathrm{CoCl}_{2} \cdot 6 \mathrm{H}_{2} \mathrm{O}, 99.3 \%\right.$ purity $)$ was purchased from MP Biomedicals, and anhydrous cobalt(II) chloride $\left(\mathrm{CoCl}_{2}\right.$, 99.4\% purity) was purchased from Wako Pure Chemical Industries. The standard samples of $\mathrm{CoO}$ and $\mathrm{LiCoO}_{2}$ were purchased from Wako Pure Chemical Industries and Kishida Chemical Company, respectively. All of the above materials were used without further purification. Samples were fixed with conductive double-sided carbon tape onto an aluminium sample holder and installed in the XAS chamber.

\subsection{Atomic multiplet calculations}

Atomic multiple simulations for $\mathrm{Co}(\mathrm{II})$ were performed using the CTM4XAS 5.0 program, including full spin-orbit coupling and crystal-field effects (Cowan, 1981; Stavitski \& Groot, 2010). The simulation default values were determined by referring to the literature concerning cobalt ions of the same oxidation state and symmetry, and optimized by repeated simulations (de Groot et al., 1993; Papaefthimiou et al., 2011).

\section{Results and discussion}

\subsection{EY-XAS measurements under normal ambient-pressure conditions}

The feasibility of EY detection under normal ambientpressure conditions was confirmed. Fig. 2 shows the XAS of 2 mm-thick $\alpha-\mathrm{Al}_{2} \mathrm{O}_{3}$ obtained using the EY-XAS (solid line) and FY-XAS (dashed line) techniques in the Al $K$-edge region. The indicated spectra were obtained by background subtraction and then normalized by the edge-jump height. One significant feature of these measurements is that the XAS can be obtained by the EY method, even though $\alpha-\mathrm{Al}_{2} \mathrm{O}_{3}$ is an insulating material. FY detection is insensitive to charging of the sample, making it applicable for measuring the XAS of insulating materials, but XAS measurements using a conventional drain-current method under vacuum conditions are inhibited by sample charging.

Here, the question of how the EY is detected by this experimental set-up arises. The EY-XAS in Fig. 2 was obtained with the same set-up as that used in the conventional sample drain-current method. Since a thick full insulator is used as the sample, direct detection of the sample draincurrent can be ruled out. Another possibility is that the positive ions are collected by the sample holder. Because the probabilities of ion desorption by soft X-ray absorption are

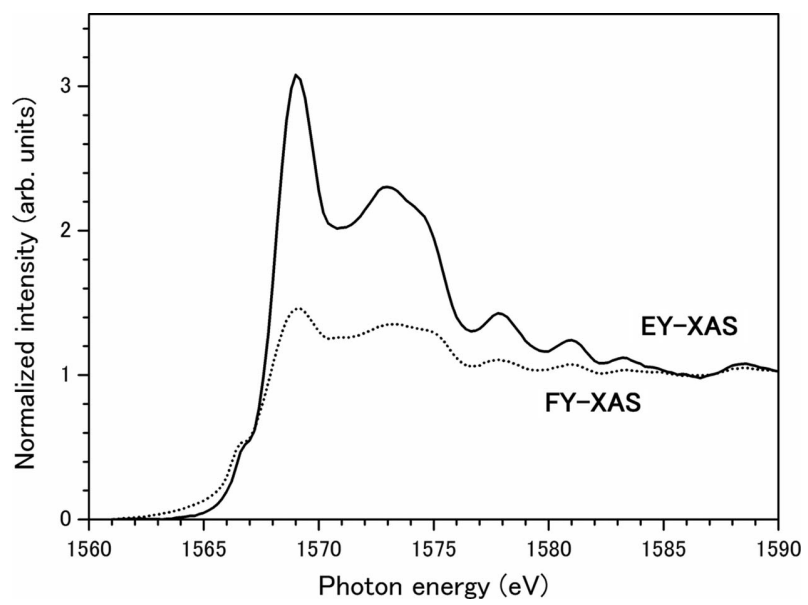

Figure 2

EY- (solid line) and FY-XAS (dashed line) of 2 mm-thick $\alpha-\mathrm{Al}_{2} \mathrm{O}_{3}$ obtained in the $\mathrm{Al} K$-edge region. Both spectra were obtained under normal ambient-pressure conditions (1 atm helium path), and were obtained by simultaneous measurements. The spectra were subjected to background subtraction and then normalized by the edge-jump height. 
negligible, the production of secondary ions is considered to be an important process. This is similar to well known processes such as conversion EY (CEY) detection in the hard X-ray region (Kordesch \& Hoffman, 1984; Tourillon et al., 1987). A conceivable EY detection scheme is as follows. Auger electrons and X-ray fluorescence, which are eliminated in the core-hole decay processes, ionize the surrounding helium gas, and secondary ions can be collected by the negatively biased sample holder. The collected ions are neutralized on the surface of the sample holder, and the number of lost electrons is measured as the drain current. In order to confirm the proposed model, the effects on the sample current of positive and negative bias voltages were investigated by changing the polarities of the dry batteries. Reversing the bias voltage reversed the polarity of the signal. This result can be interpreted as the positively biased sample holder collecting eliminated and secondary electrons.

The EY-XAS measurements of insulating samples without sample charging can also be interpreted by considering the CEY detection scheme. Soft X-ray irradiation eliminates electrons from the sample surface, and positive charges will be distributed on the sample surface. Under vacuum conditions, distortion of the spectral shape should occur as a result of the accumulation of positive charges on the sample surface. On the other hand, under low-vacuum or ambient-pressure conditions, the photoelectrons and Auger electrons emitted from the sample ionize the surrounding helium gas, and this produces several secondary electrons. These electrons are flooded around the sample surface and neutralize the positive surface charges. Similar phenomena are well known in secondary electron microscopy (SEM) measurements as lowvacuum SEM (Moncrieff et al., 1978).

The XAS obtained using two different detection modes show different spectral profiles. The peak height at the white line $(1569.0 \mathrm{eV})$ is 1.45 in the FY-XAS, whereas in the EY$\mathrm{XAS}$ it is 3.08. The clear peak intensity reduction in the FYXAS is known to be caused by the 'self-absorption effect' (Jaklevic et al., 1977; Zschech et al., 1992; Meitzner \& Fischer, 2002). Under ambient-pressure conditions, EY detection produces more reliable XAS than does FY detection. On the other hand, Nakanishi \& Ohta (2008) determined that the peak height at the white line is 5.4 by total EY (TEY) measurements using a microchannel-plate detector. The obtained peak height of the EY-XAS (3.08) is lower than those reported in earlier papers. This result suggests that the EY-XAS measurement was also affected by the saturation effect.

Zheng et al. (1997) indicated that the probing depth of the CEY method is deeper than that of the TEY method. This phenomenon, caused by the escape depth of electrons in solid materials, strongly depends on the kinetic energies of these electrons. The TEY signal is dominated by low-energy electrons with kinetic energies below about $20 \mathrm{eV}$, and the mean free paths of these electrons are shorter than $5 \mathrm{~nm}$ (Stöhr, 1992). However, these low-energy electrons cannot contribute to the production of helium ions, since the kinetic energies of most secondary electrons are not enough to ionize helium atoms [24.58 eV (Siegbahn et al., 1969)]. In CEY detection, higher-energy electrons such as Auger electrons can contribute to the production of secondary ions, and these electrons are eliminated from much deeper inside the sample. As a result, the XAS obtained by the CEY technique is more sensitive to the self-absorption effect than is that obtained by the TEY technique.

\subsection{EY-XAS measurement of dehydrated $\mathrm{CoCl}_{2}$ under various pressure conditions}

From the EY-XAS measurement of an insulating material, the contribution of the CEY signal to the EY-XAS measurement was confirmed. On the other hand, a conductive sample, which can measure the sample drain-current, should produce identical data to those obtained by TEY detection, even under ambient-pressure conditions. This assumption will be experimentally confirmed in this section.

Figs. 3(a)-3(d) show XAS of dehydrated $\mathrm{CoCl}_{2}$ samples obtained at the Co $L_{23}$-edge under various pressure conditions. To understand the spectral profiles, the XAS of a standard material $(\mathrm{CoO})$ is also shown in Fig. 3(e). Figs. 3(a) and $3(b)$ show the EY-XAS for pure $\mathrm{CoCl}_{2}$ powder under a high vacuum $\left(1 \times 10^{-5} \mathrm{~Pa}\right)$ and normal ambient atmospheric pressure conditions, respectively. The $L_{23}$-edge XAS contain two regions $\left(L_{2}\right.$ and $\left.L_{3}\right)$ arising from spin-orbit splitting of the $2 p_{3 / 2}^{-1}$ and $2 p_{1 / 2}^{-1}$ core holes. These spectra are almost identical, and the identical XAS obtained under different pressure conditions suggests two facts. The first is that the high pressure caused by the surrounding helium gas does not affect the

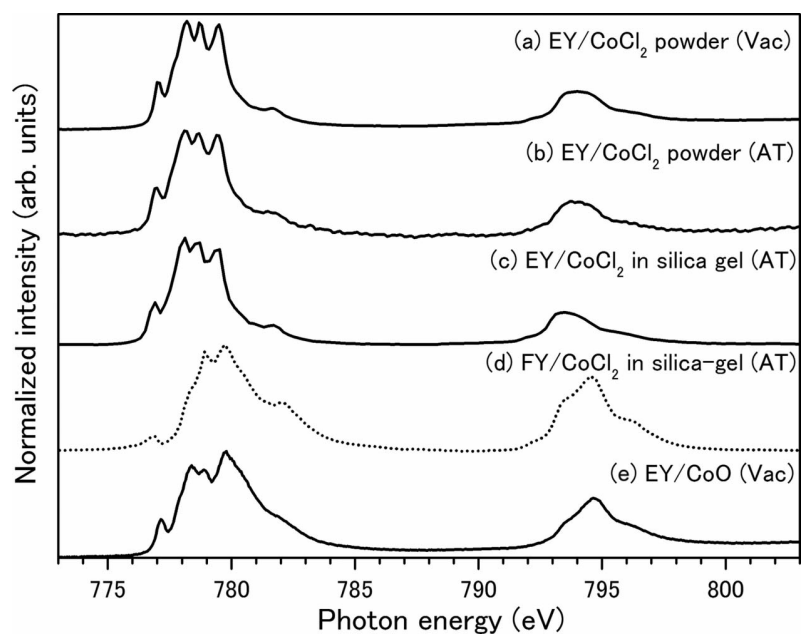

Figure 3

Co $L_{23}$-edge photoabsorption spectra of Co compounds under various pressure conditions: (a) EY-XAS of dehydrated $\mathrm{CoCl}_{2}$ powder, measured under high vacuum $\left(1 \times 10^{-5} \mathrm{~Pa}\right),(b)$ EY-XAS of dehydrated $\mathrm{CoCl}_{2}$ powder, measured under normal ambient-pressure conditions, $(c) \mathrm{EY}$ XAS of dehydrated $\mathrm{CoCl}_{2}$ in silica gel, measured under normal ambientpressure conditions, (d) FY-XAS of dehydrated $\mathrm{CoCl}_{2}$ in silica gel, measured under normal ambient-pressure conditions; the spectra in $(c)$ and $(d)$ were simultaneously observed. (e) EY-XAS of dehydrated CoO powder, measured under high vacuum as a standard material. XAS obtained by EY detection are indicated by solid lines and that by FY detection is indicated by a dashed line. 
chemical and electronic properties of the sample molecules. The second is that TEY detection is achievable for conductive samples, even under ambient-pressure conditions, as described above. As a result, the present measurements clarified that the EY-XAS under ambient-pressure conditions corresponds to the mixed data of the TEY and CEY spectra. For conductive materials, the EY-XAS measurements mainly correspond to the data obtained by TEY detection, whereas the contribution of CEY detection becomes significant for insulating materials, and is affected by the saturation effect. However, even for XAS measurements of insulating materials, the obtained EY-XAS was more reliable than the FYXAS measurements. Therefore, EY-XAS measurements are applicable in XAS measurements under ambient-pressure conditions.

Fig. 3(c) shows the EY-XAS for a colored desiccant under atmospheric pressure conditions. This spectrum is identical to the top two spectra, and suggests that $\mathrm{CoCl}_{2}$ contained in the silica gel ball is identical to the pure $\mathrm{CoCl}_{2}$ reagent. This result indicates that, although silica gel is an insulating material, the saturation effect is negligible in the present EY-XAS because of the low concentration of silica gel. Fig. 3(d) shows the FY-XAS of a colored desiccant under atmospheric pressure conditions, as a typical spectrum obtained by FY detection. The FY-XAS obtained under the top two experimental conditions were also almost identical to the FY-XAS of the colored desiccant (data not shown). The spectral profile obtained by FY detection is clearly different from that obtained by EY detection. The intensity of the $L_{3}$ band (in the 776-778 eV range) decreased in the FY-XAS. The area intensity ratio of the $L_{2}: L_{3}$ band in the EY-XAS is $1: 3.1$, whereas that of FY-XAS is $1: 1.8$. A similar phenomenon was indicated in earlier reports, and is interpreted as suppression of the $L_{3}$ band intensity by the saturation effect (Liu et al., 2007). Furthermore, depletion of some specific peaks can be confirmed in the multiplet structures. For example, the first and second peaks at 776.9 and $778.1 \mathrm{eV}$ are clearly suppressed in the FY-XAS. This phenomenon seems to arise because the fluorescence decay probabilities strongly depend on the final states (de Groot et al., 1994). The elimination of fluorescence $\mathrm{X}$-rays is expected to be suppressed on excitation of these final states. The present results also demonstrate that EY-XAS measurements are applicable even under normal ambientpressure conditions, and produce more reliable XAS data than do FY-XAS measurements.

Fig. 3(e) shows the EY-XAS of $\mathrm{CoO}$ as a reference material corresponding to dehydrous $\mathrm{CoCl}_{2}$. The XAS of anhydrous $\mathrm{CoCl}_{2}$ was found to be similar to that of $\mathrm{CoO}$, indicating that the oxidation states of the cobalt ions as well as the symmetries of the $\mathrm{CoCl}_{2}$ crystals are analogous to those of CoO. In $\mathrm{CoO}$, the $\mathrm{Co}(\mathrm{II})$ ions are coordinated to oxygen anions with octahedral geometry and $O_{h}$ symmetry (de Groot $e t$ al., 1993). This interpretation is consistent with previous $\mathrm{X}$-ray diffraction investigations of $\mathrm{CoCl}_{2}$ crystals (Grime \& Santos, 1934). The chlorine atoms adopt a cubic close-packed arrangement with the cobalt atoms located at the centers of octahedral groups of chlorine atoms.

\subsection{Comparison of EY-XAS of unhydrated and hydrated $\mathrm{CoCl}_{2}$}

Figs. 4(a) and 4(b) show the EY-XAS for a colored desiccant under normal ambient-pressure conditions. The former is the spectrum obtained for dehydrated $\mathrm{CoCl}_{2}$, which is sky blue, recorded under $1 \mathrm{~atm}$ of pure helium. The latter is the spectrum of $\mathrm{CoCl}_{2}$ hydrate, which is deep purple, recorded under $1 \mathrm{~atm}$ of a mixed helium/water gas. In order to confirm the sample conditions, photographs of the samples are shown as insets. Of particular interest are the spectral profiles of the hydrated and dehydrated $\mathrm{CoCl}_{2}$ obtained using EY-XAS, which differ substantially. The intensities of the multiplet peaks in the range $777-779 \mathrm{eV}$ decreased in the XAS of $\mathrm{CoCl}_{2} \cdot 6 \mathrm{H}_{2} \mathrm{O}$, whereas the peak at $780.5 \mathrm{eV}$ was enhanced. Since both measurements were performed under high-pressure conditions, the possibility that the high pressure changes the electronic structure of the sample can be ruled out. Another interpretation of the spectral change is a change in the valence or molecular structure as a result of hydration. The pronounced differences in the line shapes suggest that the local structures and/or the oxidation states of the cobalt ions are significantly different in the two samples.

The XAS of $\mathrm{CoCl}_{2} \cdot 6 \mathrm{H}_{2} \mathrm{O}$ and standard trivalent cobalt compounds such as $\mathrm{LiCoO}_{2}$ are very similar (Fig. 4c). At first glance, this agreement suggests that the oxidation state changed from divalent to trivalent. However, this is unlikely because of the instability of trivalent cobalt ions. Although cobalt(III) is stable in the presence of a complexing agent such as ammonia, in an aqueous solution containing no complexing agent cobalt(III) is easily reduced to cobalt(II) (Rajbir, 2002). The impurities contained in the silica gel could act as complexing agents and could affect the oxidation state of the cobalt during the hydration/dehydration processes. However,

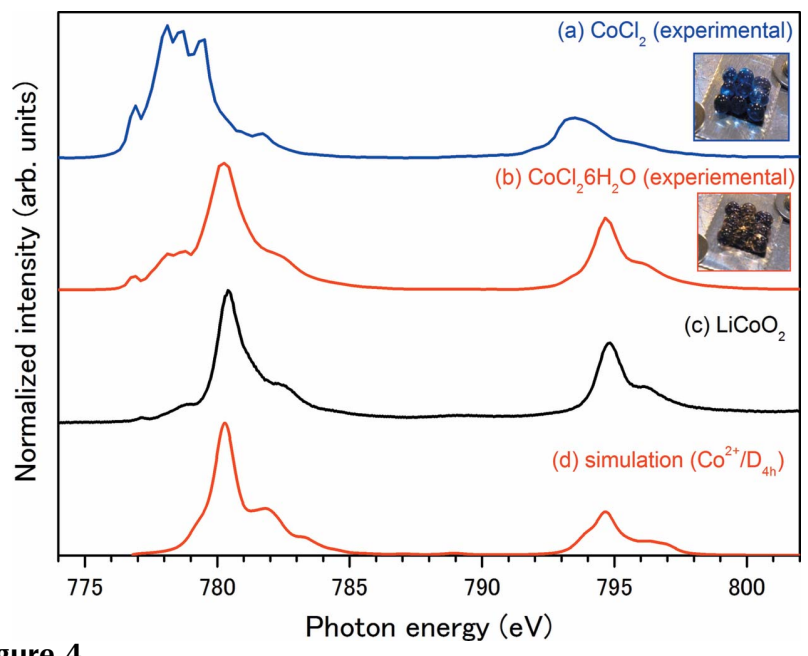

Figure 4

Co $L_{23}$-edge photoabsorption spectra of Co compounds under various pressure conditions: (a) EY-XAS of dehydrated $\mathrm{CoCl}_{2}$ in silica gel, measured under $1 \mathrm{~atm}$ helium condition, $(b)$ EY-XAS of hydrated silica gel (i.e. $\mathrm{CoCl}_{2} \cdot 6 \mathrm{H}_{2} \mathrm{O}$ ), measured under $1 \mathrm{~atm}$ helium/water mixture conditions, (c) EY-XAS of $\mathrm{LiCoO}_{2}$ powder, measured under high vacuum, as a standard material, and $(d)$ spectrum from ligand-field multiplet simulation of cobalt(II) ions with $D_{4 h}$ symmetry. 
this scenario can be ruled out because the change in the spectral profile was replicated by XAS analysis of pure $\mathrm{CoCl}_{2}$ (data not shown). Therefore, we conclude that it is unlikely that water molecules added by hydration oxidized the cobalt ions in $\mathrm{CoCl}_{2} \cdot 6 \mathrm{H}_{2} \mathrm{O}$.

Another interpretation is that the crystal symmetry is changed by hydration. $\mathrm{X}$-ray diffraction studies suggested the following crystal structure for $\mathrm{CoCl}_{2} \cdot 6 \mathrm{H}_{2} \mathrm{O}$ (Mizuno et al., 1959; Mizuno, 1960; El Saffar, 1960; Waizumi et al., 1990; Souissi \& Kammoun, 2011): two $\mathrm{Cl}^{-}$ions and four water molecules are coordinated to a $\mathrm{Co}^{2+}$ ion in an octahedral geometry to form a $\mathrm{CoCl}_{2} \cdot 4 \mathrm{H}_{2} \mathrm{O}$ group, whereas the other two water molecules of the formula unit are relatively free, and the groups are joined to one another by hydrogen bonds $(\mathrm{O} \cdots \mathrm{H}-\mathrm{O}-\mathrm{H} \cdots \mathrm{O}$ and $\mathrm{O}-\mathrm{H} \cdots \mathrm{Cl})$. The $\mathrm{CoCl}_{2} \cdot 6 \mathrm{H}_{2} \mathrm{O}$ crystal has a quasi-octahedral geometry, and the complex unit belongs to the point-group $D_{4 h}$ symmetry. Based on these $\mathrm{X}$-ray diffraction reports, we can assume that the symmetry of the $\mathrm{CoCl}_{2}$ crystal changed from $O_{h}$ to $D_{4 h}$ as a result of hydration.

This hypothesis has been confirmed by ligand-field multiplet simulation (de Groot et al., 1993; Papaefthimiou et al., 2011). Fig. $4(d)$ shows the simulated spectra, assuming a $D_{4 h}$ symmetry with a ligand-field strength of $10 \mathrm{Dp} / \mathrm{dt} / \mathrm{ds}=1.2 /$ $-0.5 / 0.1 \mathrm{eV}$ for the divalent cobalt ion. The simulations faithfully reproduce the main spectral line profiles, whereas the spectrum from the simulation does not reproduce the lower-energy sides of the $L_{2}$ and $L_{3}$ bands. The discrepancy can be interpreted as contamination of the dehydrated $\mathrm{CoCl}_{2}$ signal. For example, the small peaks observed in the range 777-779 eV could not be reproduced in the simulated spectrum. However, these peaks correspond to the first three peaks observed in the spectrum of dehydrated $\mathrm{CoCl}_{2}$ (Fig. 4a). Therefore, the discrepancy can be interpreted as partly dried $\mathrm{CoCl}_{2}$ being contained in the colored silica gel.

Furthermore, our explanation is also strengthened by the repeatable nature of the XAS analysis. After measurement of a hydrated sample, we stopped the helium/water mixed gas flow. This procedure evacuated the sample region and the $\mathrm{CoCl}_{2}$ underwent dehydration. Dehydration of the sample can be unambiguously confirmed by the change in the sample color (a color change from purple to blue). When we performed XAS measurements on this dehydrated sample, the spectrum indicated that the sample possessed $O_{h}$ symmetry. Once the sample was rehydrated by immersion in water (undergoing a color change from blue to purple), subsequent XAS analysis revealed the $D_{4 h}$ nature of the analyte. The repeatable change in the XAS is achieved through the hydration and dehydration reactions alone, without adding a special oxidizing or reducing agent. Therefore, although the XAS of $\mathrm{CoCl}_{2} \cdot 6 \mathrm{H}_{2} \mathrm{O}$ mimics the XAS of standard trivalent materials, we can eliminate the possibility that the spectral changes originate from a change in the oxidation state of the cobalt ion. We can conclude that the valence of the cobalt ion in $\mathrm{CoCl}_{2} \cdot 6 \mathrm{H}_{2} \mathrm{O}$ is divalent and the crystal has $D_{4 h}$ symmetry.

\section{Conclusions}

In the present study, it was demonstrated that EY soft XAS under normal ambient-pressure conditions can be applied by using the same layout as that with a normal sample draincurrent used in conventional soft X-ray analysis. The feasibility of this method was confirmed by XAS measurements of $\mathrm{Al}_{2} \mathrm{O}_{3}$. EY XAS can be understood to have the mixed data of the total EY and conversion EY spectra. The drawback of EYXAS under ambient conditions is that the obtained spectrum contains CEY signals. The CEY signals are enhanced on the measurement of insulating materials, and this causes spectral suppression as a result of the self-absorption effect. Despite this drawback, the obtained spectrum was more reliable than that using FY-XAS measurements, and is applicable in XAS measurements under ambient-pressure conditions.

EY-XAS analysis was applied to the analysis of cobalt ions in anhydrous $\mathrm{CoCl}_{2}$ crystals and to hydrated $\mathrm{CoCl}_{2}$ crystals under normal ambient-pressure conditions. The EY-XAS results unambiguously distinguished the chemical states of the cobalt ions in each sample. XAS simulation was used to show that the $\mathrm{CoCl}_{2}$ crystal underwent an octahedral/quasi-octahedral structural change by a hydration/dehydration process. We have successfully demonstrated that EY-XAS analysis under ambient pressure, using a helium path, is applicable to chemical state analysis, even for hydrated samples. This represents a significant milestone in the development of XAS analysis using soft $\mathrm{X}$-rays under ambient conditions.

This work was supported by the GIGNO project at SPring8. The author gratefully thanks the members of the GIGNO committee for useful advice concerning this study. This work was financially supported by a Grant-in-Aid for challenging Exploratory Research (22655023). This study was conducted with the approval of the SPring-8 Proposal Review Committee (Proposals 2010A1104, 2010B1198, 2011B1993 and 2012A1847).

\section{References}

Cowan, R. D. (1981). The Theory of Atomic Structure and Spectra. Berkeley: University of California Press.

El Saffar, Z. M. (1960). J. Phys. Soc. Jpn, 17, 1334-1337.

Grime, H. \& Santos, J. A. (1934). Z. Kristallogr. 88, 136-141.

Groot, F. de, Arrio, M., Sainctavit, P., Cartier, C. \& Chen, C. (1994). Solid State Commun. 92, 991-995.

Groot, F. M. de, de Smit, E., van Schooneveld, M. M., Aramburo, L. R. \& Weckhuysen, B. M. (2010). ChemPhysChem, 11, 951-962.

Groot, F. M. F. de, Abbate, M., Elp, J. v., Sawatzky, G. A., Ma, Y. J., Chen, C. T. \& Sette, F. (1993). J. Phys. Condens. Matter, 5, 22772288.

Groot, F. M. F. de \& Kotani, A. (2008). Core Level Spectroscopy of Solids. Boca Raton: CRC Press.

Guo, J.-H., Augustsson, A., Englund, C.-J. \& Nordgren, J. (2004). AIP Conf. Proc. 705, 1066-1069.

Huse, N., Kim, T. K., Jamula, L., McCusker, J. K., de Groot, F. M. \& Schoenlein, R. W. (2010). J. Am. Chem. Soc. 132, 6809-6816.

Jaklevic, J., Kirby, J., Klein, M., Robertson, A., Brown, G. \& Eisenberger, P. (1977). Solid State Commun. 23, 679-682.

Kolmakov, A., Dikin, D. A., Cote, L. J., Huang, J., Abyaneh, M. K., Amati, M., Gregoratti, L., Günther, S. \& Kiskinova, M. (2012). Nat. Nanotechnol. 6, 651-657. 
Kordesch, M. \& Hoffman, R. (1984). Phys. Rev. B, 29, 491-492.

Liu, H., Guo, J., Yin, Y., Augustsson, A., Dong, C., Nordgren, J., Chang, C., Alivisatos, P., Thornton, G., Ogletree, D. F., Requejo, F. G., de Groot, F. \& Salmeron, M. (2007). Nano Lett. 7, 19191922.

Meitzner, G. \& Fischer, D. (2002). Microchem. J. 71, 281-286.

Mizuno, J. (1960). J. Phys. Soc. Jpn, 15, 1412-1420.

Mizuno, J., Ukei, K. \& Sugawara, T. (1959). J. Phys. Soc. Jpn, 14, 383.

Moncrieff, D. A., Robinson, V. N. E. \& Harris, L. B. (1978). J. Phys. D, 11, 2315-2325.

Nakanishi, K. \& Ohta, T. (2008). Mem. SR Cent. Ritsumeikan Univ. 10, 105.

Nakanishi, K., Yagi, S. \& Ohta, T. (2010). AIP Conf. Proc. 1234, 931934.

Ohashi, H., Ishiguro, E., Tamenori, Y., Okumura, H., Hiraya, A., Yoshida, H., Senba, Y., Okada, K., Saito, N., Suzuki, I., Ueda, K., Ibuki, T., Nagaoka, S., Koyano, I. \& Ishikawa, T. (2001). Nucl. Instrum. Methods Phys. Res. A, 467, 533-536.

Papaefthimiou, V., Dintzer, T., Dupuis, V., Tamion, A., Tournus, F., Hillion, A., Teschner, D., Hävecker, M., Knop-Gericke, A., Schlögl, R. \& Zafeiratos, S. (2011). ACS Nano, 5, 2182-2190.

Rajbir, S. (2002). Inorganic Chemistry. Transition Metals. New Delhi: Mittal.

Roper, M. D., van der Laan, G., Flaherty, J. V. \& Padmore, H. A. (1992). Rev. Sci. Instrum. 63, 1482.

Schreck, S., Gavrila, G., Weniger, C. \& Wernet, P. (2011). Rev. Sci. Instrum. 82, 103101.
Siegbahn, K., Nordling, C., Johansson, G., Hedman, J., Heden, P. F., Hamrin, K., Gelius, U., Bergmark, T., Werme, L. O., Manne, R. \& Baer, Y. (1969). ESCA Applied to Free Molecules. New York: Elsevier.

Smit, E. de, Swart, I., Creemer, J. F., Hoveling, G. H., Gilles, M. K., Tyliszczak, T., Kooyman, P. J., Zandbergen, H. W., Morin, C., Weckhuysen, B. M. \& de Groot, F. M. (2008). Nature (London), 456, 222-225.

Solomon, M. E. (1945). Ann. Appl. Biol. 32, 75-85.

Souissi, H. \& Kammoun, S. (2011). Mater. Sci. Appl. 2, 1121-1126.

Stavitski, E. \& de Groot, F. M. (2010). Micron, 41, 687-694.

Stöhr, J. (1992). NEXAFS Spectroscopy. Berlin: Springer.

Tamenori, Y. (2010). J. Synchrotron Rad. 17, 243-249.

Tanaka, T., Maréchal, X.-M., Hara, T., Tanabe, T. \& Kitamura, H. (1998). J. Synchrotron Rad. 5, 459-461.

Tourillon, G., Dartyge, E., Fontaine, A., Lemonnier, M. \& Bartol, F. (1987). Phys. Lett. A, 121, 251-257.

Waizumi, K., Masuda, H., Ohtaki, H., Tsukamoto, K. \& Sunagawa, I. (1990). Bull. Chem. Soc. Jpn, 63, 3426-3433.

Yagi, S., Matsumura, Y., Soda, K., Hashimoto, E. \& Taniguchi, M. (2004). Surf. Interface Anal. 36, 4-1066.

Zaanen, J., Sawatzky, G., Fink, J., Speier, W. \& Fuggle, J. (1985). Phys. Rev. B, 32, 4905-4913.

Zheng, S., Hayakawa, S. \& Gohshi, Y. (1997). J. Electron Spectrosc. Relat. Phenom. 87, 81-89.

Zschech, E., Tröger, L., Arvanitis, D., Michaelis, H., Grimm, U. \& Baberschke, K. (1992). Solid State Commun. 82, 1-5. 